\title{
Dynamical fluctuations in biochemical reactions and cycles
}

\author{
S. Pressé, ${ }^{1}$ K. Ghosh, ${ }^{2}$ R. Phillips, ${ }^{3}$ and K. A. Dill ${ }^{1}$ \\ ${ }^{1}$ Department of Pharmaceutical Chemistry, University of California, San Francisco, California 94158, USA \\ ${ }^{2}$ Department of Physics and Astronomy, University of Denver, Denver, Colorado 80208, USA \\ ${ }^{3}$ Department of Applied Physics, California Institute of Technology, Pasadena, California 91125, USA \\ (Received 30 October 2009; revised manuscript received 13 July 2010; published 15 September 2010)
}

\begin{abstract}
We develop theory for the dynamics and fluctuations in some cyclic and linear biochemical reactions. We use the approach of maximum caliber, which computes the ensemble of paths taken by the system, given a few experimental observables. This approach may be useful for interpreting single-molecule or few-particle experiments on molecular motors, enzyme reactions, ion-channels, and phosphorylation-driven biological clocks. We consider cycles where all biochemical states are observable. Our method shows how: (1) the noise in cycles increases with cycle size and decreases with the driving force that spins the cycle and (2) provides a recipe for estimating small-number features, such as probability of backward spin in small cycles, from experimental data. The back-spin probability diminishes exponentially with the deviation from equilibrium. We believe this method may also be useful for other few-particle nonequilibrium biochemical reaction systems.
\end{abstract}

DOI: 10.1103/PhysRevE.82.031905

PACS number(s): 87.16.dj, 82.39.Rt, 87.10.Ca, 87.18.Tt

\section{INTRODUCTION}

We are interested in noise in chemical cycles that are driven and out-of-equilibrium. Consider a cycle of $s$ different conformational states, for example of a protein. Some such cycles are driven to "spin" in a "forward" direction, for example by ATP hydrolysis. Examples include molecular motors, such as the $F_{0} F_{1}$ ATP synthase [1]; the HSP90 chaperone protein complex, which assists in protein folding $[2,3]$; and circadian clock circuits, driven by changing phosphorylation states [4]. The dynamics are noisy because of small particle numbers.

The kinetics of biochemical reactions are commonly treated using master equations or continuum Fokker-Planck equations [5]. In those approaches, the central quantities are the full time-dependent particle distribution functions as well as point statistics and correlations derived from the particle distribution [6]. In contrast to master equations, we present a method for treating small-system dynamics that centers on ensembles of trajectories, rather than on particle distribution functions [13]. Figure 1 shows an example of a singleparticle trajectory in a three-state system. We compute the stochastic dynamics using a trajectory-based approach called maximum caliber (MaxCal). First introduced by E. T. Jaynes [7-9], MaxCal computes the ensemble of trajectories taken by the system as follows [10,11]: (1) A dynamical partition function $Q$ is expressed as a sum over all the possible microscopic trajectories, (2) each trajectory has a statistical weight depending on the states through which it passes, but these statistical weights are unknown a priori, (3) experimentally measurable quantities are expressed as averages over those trajectories, (4) using measured first moments of fluxes, we find the undetermined statistical weights, and thus $Q$, then (5) other properties of the system are computed directly from $Q$, often as simple derivatives. In building our trajectory ensemble, we assume that all transitions between states are observable [12]; we do not model systems having hidden states. MaxCal is, in principle, a powerful tool for modeling stochastic dynamics, particularly for single-particle and few-particle systems far from equilibrium. MaxCal is to dynamical problems what maximum entropy is for problems of equilibrium statistical mechanics and the method has been validated in some simple experimental situations $[14,15]$.

\section{MAXCAL APPROACH: A SIMPLE 2-STATE DIFFUSION EXAMPLE}

First, to illustrate $\mathrm{MaxCal}$, we consider a simple problem of two-state Markovian diffusion with state labels 0 and 1 $[10,15]$. Initially, $N_{0}$ particles are in state 0 and $N_{1}$ particles in state 1. To keep the counting discrete, we partition time into small intervals of length $\delta t$. Within $\delta t$, each particle may either stay in its present state or jump to the other. A microscopic system trajectory $\Gamma$ is one particular possible time trace of $N$ such steps, over a total time $N \delta$ t. As such, each trajectory considered here is a discrete time Markovian process. During that time course, a particular trajectory, $\Gamma$, will have $N_{10, \Gamma}$ transitions from 0 to $1, N_{01, \Gamma}$ from 1 to $0, N_{00, \Gamma}$ from 0 to 0 and $N_{11, \Gamma}$ from 1 to 1 . The dynamical partition function $Q$ is the sum of statistical weights over all possible trajectories,

$$
Q=\sum_{\Gamma} \sigma_{00}^{N_{00, \Gamma}} \sigma_{11}^{N_{11, \Gamma}} \sigma_{10}^{N_{10, \Gamma}} \sigma_{01}^{N_{01, \Gamma}}=\sum_{\Gamma} p_{\Gamma},
$$

where $\sigma_{i j}$ with $(i, j)=0$ or 1 are the four statistical weights for the corresponding types of transitions; they are undetermined at first. Experimentally observable average quantities, $\left\langle N_{i j}\right\rangle$, are used to parametrize the set $\left\{\sigma_{i j}\right\}$ appearing in $Q$ using the following recipe [10]
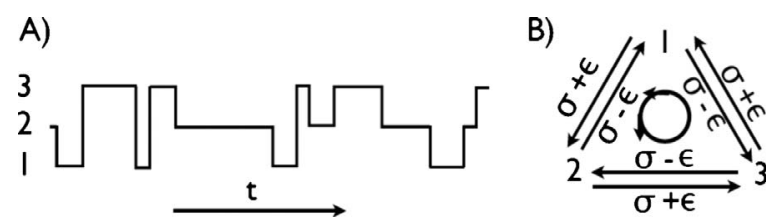

FIG. 1. (a) One possible time trajectory for a three-state cycle. (b) A three-state cycle, showing the definitions of rates used below. 


$$
\left\langle N_{i j}\right\rangle=\frac{\partial \ln Q}{\partial \ln \sigma_{i j}} .
$$

Our strategy throughout will be to compute other properties or cumulants of the memoryless discrete time dynamics by taking appropriate derivatives of Eq. (1). Appendix A briefly discusses the input constraints to MaxCal.

For multiple independent particles, the system dynamical partition function factorizes into

$$
Q=q_{0}^{N_{0}} q_{1}^{N_{1}}
$$

where $q_{i}$, the dynamical partition function for individual particles originating at state $i$, is

$$
q_{i}=\left(\begin{array}{ll}
1 & 1
\end{array}\right) \mathbf{G}^{N}\left(\begin{array}{l}
\delta_{i, 0} \\
\delta_{i, 1}
\end{array}\right)
$$

and the column vector on the right hand side of the above specifies the system's initial condition while $\mathbf{G}$ is a transition matrix with matrix elements $G_{i j}=\sigma_{i j}$ with $i, j=0,1$. For a single time step, $N=1$, we have

$$
Q=\left(\sigma_{00}+\sigma_{10}\right)^{N_{0}}\left(\sigma_{11}+\sigma_{01}\right)^{N_{1}} .
$$

The approach is to obtain from experiments the average forward and backward fluxes from state 0 to 1 and 1 to 0 , $\left\langle J_{10}\right\rangle=\partial \ln Q / \partial \ln \sigma_{10}$ and $\left\langle J_{01}\right\rangle=\partial \ln Q / \partial \ln \sigma_{01}$, respectively. From those, we obtain $\sigma_{10}$ and $\sigma_{01}$ using the following two equations

$$
\left\langle N_{i j}\right\rangle \equiv\left\langle J_{i j}\right\rangle=N_{j} \sigma_{i j} /\left(\sigma_{i j}+\sigma_{j j}\right) .
$$

These two equations fully determine $Q$ since $\sigma_{00}$ and $\sigma_{11}$ are eliminated using $N_{0}=\left\langle N_{00}\right\rangle+\left\langle N_{10}\right\rangle$ and $N_{1}=\left\langle N_{11}\right\rangle+\left\langle N_{01}\right\rangle$ yielding $\sigma_{00}=1-\sigma_{10}$ and $\sigma_{11}=1-\sigma_{01}$. In this case, $Q=1$ and $\sigma_{10}$ and $\sigma_{01}$ are jump probabilities.

The average net flux from 0 to 1 , defined as $\langle J\rangle \equiv\left\langle J_{10}-J_{01}\right\rangle$, is

$$
\langle J\rangle=\sigma_{10} N_{0}-\sigma_{01} N_{1} .
$$

The fluctuations, $\left\langle J^{2}\right\rangle_{c}=\left\langle J^{2}\right\rangle-\langle J\rangle^{2}$ (where $c$ is for cumulant), are given by

$$
\left\langle J^{2}\right\rangle_{c}=\sigma_{10}\left(1-\sigma_{10}\right) N_{0}+\sigma_{01}\left(1-\sigma_{01}\right) N_{1} .
$$

Microfluidics experiments have confirmed the validity of these expressions for symmetrical diffusion processes where $\sigma_{01}=\sigma_{10}[14]$.

\section{TREATING OTHER REACTION TOPOLOGIES}

\section{A. Forced diffusion in a linear string of states}

Now, we generalize beyond two states to illustrate the methods we will need in later sections. Consider a long linear string of interconnected identical states, $\ldots \leftrightarrow-1 \leftrightarrow 0 \leftrightarrow 1 \ldots$, as occurs in linear molecular motors. We first express the two unknown statistical weights in terms of two variables $\sigma$ and $\mathrm{v}$,

$$
\sigma_{10} \equiv \sigma+\mathrm{v} / 2 ; \quad \sigma_{01} \equiv \sigma-\mathrm{v} / 2 .
$$

This change of variables is convenient because $\sigma$ corresponds to a directionless diffusion coefficient and $\mathrm{v}$ corre- sponds to the average drift velocity that would arise in a diffusion equation with drift (Smoluchowski equation). For small $\delta t$ the average net instantaneous flux between any two states, say 0 and 1 , is $\langle J\rangle=(\mathrm{v} / 2)\left(N_{0}+N_{1}\right)+\sigma\left(N_{0}-N_{1}\right)$ and the fluctuations are $\left\langle J^{2}\right\rangle_{c}=(\mathrm{v} / 2)\left(N_{0}-N_{1}\right)+\sigma\left(N_{0}+N_{1}\right)$. Invoking particle conservation at state 0 , we find

$$
N_{0}(t+\delta t)-N_{0}(t)=\sigma\left(N_{-1}-2 N_{0}+N_{1}\right)+\mathrm{v} / 2\left(N_{-1}-N_{1}\right),
$$

which reduces, in the limit of small steps and many states, to the Smoluchowski equation,

$$
\frac{\partial c}{\partial t}=-\nu \frac{\partial c}{\partial x}+D \frac{\partial^{2} c}{\partial x^{2}},
$$

where $c$ is the particle number at location or site $x$, the drift velocity is $\nu \equiv(\delta x / \delta t) \mathrm{V}$ and $D \equiv\left(\delta x^{2} / \delta t\right) \sigma{ }^{1}$ This simply shows that the Caliber approach reduces, as it should, to Smoluchowski equation, and is not unexpected $[16,17]$. For example, by constraining both the average flux and average energy, R. M. L. Evans derives the Fokker-Planck equation for driven systems and shows how equilibrium detailedbalance conditions are modified by rare subensembles of trajectories exhibiting net flux after some long time $\tau$ [17].

\section{B. Dynamics of three cycles}

In MaxCal, the 3-state cycle is a straightforward generalization of the two-state problem. In this case, 6 hopping fluxes from state $i$ to $j,\left\langle N_{i \rightarrow j}\right\rangle$, are obtained by averaging over repeated experiments or, in MaxCal, averaged over the trajectory ensemble. The indices $i$ and $j$ are equal to 0,1 , or 2 , so we have 6 constraints [15]. While studying a system with 6 arbitrary rate coefficients gives little general insight, we illustrate here our general results by focusing instead on a subproblem characterized by two rates. All three forward rates are $\sigma+\epsilon$ and all three backward rates are $\sigma-\epsilon . \sigma$ is an intrinsic rate identical in the forward and backward directions and $\epsilon$ is the degree to which detailed balance is broken (which need not be small). We believe this is among the simplest out-of-equilibrium systems having a small number of parameters. For example, in biology, large $\epsilon$ may represent a high ATP concentration. Following the MaxCal procedure above, we find that in the $1 \leftrightarrow 2$ branch of the three cycle, the average net flux, $J=N_{21}-N_{12}$, and flux fluctuations are

$$
\langle J\rangle=(1+2 N \epsilon) / 3
$$

$$
\left\langle J^{2}\right\rangle_{c}=\frac{2 N}{9}\left(\sigma-2 \epsilon^{2}\right),
$$

where the net average flux is the average number of particles going from sites 1 to 2 from which we subtract the number

\footnotetext{
${ }^{1}$ For a small enough time interval $\delta t$, the ratio $\sigma / \delta t$ is small but finite. As $\delta t$ is reduced, the probability of jump $\sigma$ shrinks while $\sigma / \delta t$ remains constant. In other words, the factor of $\sigma$ appears in $D=\left(\delta x^{2} / \delta t\right) \sigma$ because of the arbitrariness in selecting the size of our time window. We may remove this arbitrariness by fixing $\delta t$ to $\delta t \times \sigma$ so that $D$ is now equivalent to the usual diffusion coefficient.
} 
going from sites 2 to 1 within interval of length $N \delta t$. In the presence of a single particle at site $1($ at $t=0)$, for an undriven system, $\epsilon=0$, in the long-time limit a net particle exchange will have occurred between states 1 and 2. From Eq. (12) we see that when $\epsilon=0$, or all rates are the same, the net particle exchange from site 1 to 2 is $1 / 3$, and that from 1 to 3 is $1 / 3$ as well. Also, under such conditions, the fluctuations are unbounded in time, $\left\langle J^{2}\right\rangle_{c} /\langle J\rangle \sim \mathrm{O}(N)$. This is because the number of transitions $1 \rightarrow 2$ increases linearly with time $N$. However, the ratio $\left\langle J^{2}\right\rangle_{c} /\langle J\rangle$ becomes bounded as the system is driven away from equilibrium.

\section{Dynamics of $s$-state cycles}

Now, consider a cyclic network with $s$ states. Again, we express the statistical weights in the forward direction as $\sigma+\epsilon$ and the backward direction as $\sigma-\epsilon$. MaxCal gives as the average flux and the fluctuations (in the long-time limit, $N \rightarrow \infty)$,

$$
\langle J\rangle=\frac{2 N}{s} \epsilon \text { and }\left\langle J^{2}\right\rangle_{c}=\frac{2 N}{s^{2}}\left(\sigma-2 \epsilon^{2}\right) .
$$

We compute a noise-to-signal quantity $\delta_{s}$ for an arbitrary network of $s$ states as

$$
\delta_{s}=\frac{\left\langle J^{2}\right\rangle_{c}}{\langle J\rangle^{2}}=\frac{1}{N}\left(\frac{\sigma}{2 \epsilon^{2}}-1\right) .
$$

It is useful to express our results in terms of $r$, a constant ratio of forward to backward rates,

$$
r=\left(\frac{\sigma+\epsilon}{\sigma-\epsilon}\right)^{s} .
$$

This also means that in order to hold $r$ fixed for any cycle size, i.e., same driving force for any cycle size, we need to rescale $\epsilon$ as follows

$$
\epsilon(r)=\sigma\left(1-\frac{2}{r^{1 / s}+1}\right) .
$$

Since, from Eq. (16), increasing the number of states $s$ decreases the value of $\epsilon$, the noise $\delta_{s}$ increases for fixed values of $r$ and $\sigma$. The main conclusion is that, for our particular Markov site model, the noise, $\delta_{s}$, in an $s$ cycle, increases with the number of states, $s$, in the cycle, and decreases the harder the cycle is driven (i.e., greater $r$ ). We illustrate this quantitatively by solving Eqs. (14) and (16) numerically and depict the result for different $s$ and $\epsilon$ in Fig. 2. An interesting biological implication is that there may be optimal sizes for biochemical cycles. On the one hand, larger cycles may be useful for performing particular complex biochemistry, but keeping cycles smaller reduces noise. It is not clear whether there are such evolutionary forces.

Above, we have computed only averages and variances of the flux. We are also interested in the full distribution of rates with the intent of deriving a flux fluctuation ratio. A recent approach to expressing the magnitudes of nonequilibrium fluctuations has been through fluctuation theorems, either for continuous systems [18-21] or for systems with discrete chemical states [22-26], of interest here. Our method does

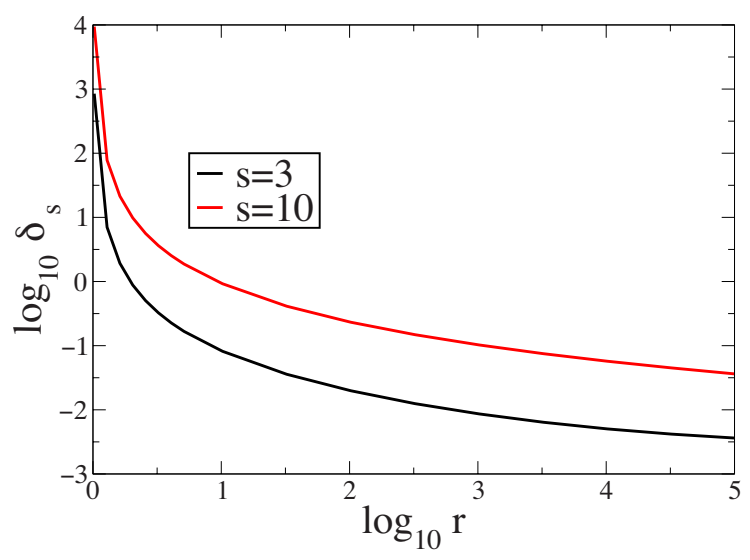

FIG. 2. (Color online) $\delta_{s}$, the ratio of flux variance to average flux squared of a $s$-state cycle as a function of $r$, the driving force to spin the cycle, for cycle sizes $s=3$ (lower curve) and $s=10$. In the figure we use $\sigma=0.4$ and $N=100$. The particular parameters are selected to illustrate features of out-of-equilibrium system. See The dynamics of three-cycles for more details.

not require near-equilibrium assumptions [27], and in principle, the method of MaxCal applies equally well to nonMarkovian dynamics $[10,16]$ and systems having spatial correlations. In contrast to other nonequilibrium relations, such as those dealing with work [28,29] or entropy [30-32], our fluctuation ratio is expressed in terms of a simple directly measurable quantity, the flux. ${ }^{2}$

\section{FLUX-FLUCTUATION RATIO}

In the spirit of earlier flux fluctuation ratios $[11,14]$, we write a ratio, $P(J ; N \delta t) / P(-J ; N \delta t)$ where $P(J ; N \delta t)$ is the probability of observing net flux $J$ over a total time of $N \delta t$. For multistate systems, we are interested in the net flux $J_{i j}$ from state $j$ to $i$ during a time interval $N \delta t$; that is, the number of particles going from $j$ to $i$ from which we subtract the number going from $i$ to $j$. The distribution function $P\left(J_{i j} ; N \delta t\right)$ is

$$
P\left(J_{i j} ; N \delta t\right)=Q^{\prime} / Q
$$

where $Q^{\prime}$ is the sum over microtrajectories that specifically have net flux $J_{i j}$ between states $i$ and $j$, where $Q$ is

$$
Q=\prod_{m} q_{m}^{N_{m}}
$$

and where $q_{m}$ is the dynamical partition function for state $m$ with $N_{m}$ particles initially. We can write the restricted sum $Q^{\prime}$ which includes only those trajectories having net flux $J_{i j}$ between states $i$ and $j$ as follows:

\footnotetext{
${ }^{2}$ In the limit of many particles, the functional form of our fluxfluctuation ratio derives from the limiting distribution of trajectories, which also follows from the central limit theorem.
} 


$$
Q^{\prime}=\prod_{m \neq i, j} q_{m}^{N_{m}} \oint \frac{d z}{2 \pi i} z^{J_{i j}-1} \widehat{q}_{i}^{N_{i}} \widehat{q}_{j}^{N_{j}},
$$

where the loop, which circles the origin, collapses all microtrajectories in the dynamical partition function for which the flux differs from $J_{i j}, \hat{q}_{i} \equiv q_{i}\left(\sigma_{j i} z^{-1}, \sigma_{i j} z\right)$ and the flux distribution characteristic function is $\widehat{q}_{i}{ }^{N} \widehat{q}_{j}{ }^{N_{j}}$, see supplementary materials for more details. When there are more than a few particles, this flux characteristic function is approximately Gaussian. In that case, by substituting $z=\exp (i \phi)$ the treatment of the integral is standard [33] and the dominant contributions are near $\phi \sim 0$, leading to

$$
\frac{P\left(J_{i j} ; N \delta t\right)}{P\left(-J_{i j} ; N \delta t\right)}=\exp \left(\frac{2\left\langle J_{i j}\right\rangle J_{i j}}{\left\langle J_{i j}^{2}\right\rangle_{c}}\right) .
$$

This flux-fluctuation ratio holds for any state topology, including cycles in any forcing regime for arbitrary time when all states are accounted for. For cycles, it says that "backwards" fluctuations become exponentially less probable with increasing "spin rate" $\left\langle J_{i j}\right\rangle$. By backward, we mean situations in which a cycle transiently spins counterclockwise if there is a net driving force to spin clockwise resembling situations described in terms of "Maxwell's Demon" or "bad actors" [11]. When $N=1$, and the flux is between two states $i$ and $j$ the mean net flux and standard deviations are given by Eqs. (7) and (8) while mean net flux and standard deviations for arbitrary $N$ are

$$
\begin{aligned}
\left\langle J_{i j}\right\rangle & =\frac{\left(\sigma_{10} N_{0}-\sigma_{01} N_{1}\right)\left(1-\left(1-\sigma_{10}-\sigma_{01}\right)^{N}\right)}{\sigma_{10}+\sigma_{01}} \\
\left\langle J_{i j}^{2}\right\rangle_{c}= & \left\{\sigma_{10} \sigma_{01}\left(N_{0}+N_{1}\right)+\left[1-\left(1-\sigma_{10}-\sigma_{01}\right)^{N}\right]\right. \\
& \left.\times\left(N_{0} \sigma_{10}^{2}+N_{1} \sigma_{01}^{2}\right)\right\} \frac{\left[1-\left(1-\sigma_{10}-\sigma_{01}\right)^{N}\right]}{\left(\sigma_{10}+\sigma_{01}\right)^{2}} .
\end{aligned}
$$

\section{DISCUSSION}

We describe here nonequilibrium fluctuations in linear and cyclic chemical reactions using the approach of maximum caliber. MaxCal quantifies the rate at which fluctuations increase with the number of states and decrease with $r$, the driving force away from equilibrium. For example, molecular motors driven at high speed should have small flux fluctuations. We believe this approach may be useful, for a broad range of systems, for exploring how noise and fluctuations in small biochemical systems can be predicted from readily computed experimental observables.

\section{ACKNOWLEDGMENTS}

We appreciate the support from NIH (Grant No. GM34993) to K.D. S.P. thanks the FQRNT for its financial support as well as J. van Drie and G. Peterson for their insights. K.G. acknowledges an FRF Grant from DU for financial support.

\section{APPENDIX A}

We remark here on our choice of constraints for MaxCal. As constraints, we use first-moment quantities such as $\left\langle N_{i j}\right\rangle$.
The number of constraints that are needed to solve a MaxCal problem depends on the number of independent statistical weights that must be determined for a given model. We could have used combinations of sums and differences of first moments. Sums and differences would be represented by the corresponding changes in Eq. (2) and would lead to the same set of statistical weights. As long as the constraints involve some linear combination of first-moment quantities, the choice of what to constrain is simply a matter of convenience. The general three-state-cycle problem we discussed would require three forward and three backward rate coefficients, and therefore would require six constraints in Max $\mathrm{Cal}$. However, we have illustrated our method on a simpler problem that requires only one forward and one backward rate constant, hence the two constraints in which we fix $\sigma$ and $\epsilon$. In addition, we note that our first-moment constraints lead to the correct master equation formulation of the dynamics considered.

Could we have used higher-moment constraints, such as $\left\langle N_{i j}^{2}\right\rangle$ instead of first-moment constraints? Following Gibbs [35] and others [34], we note that constraints must be extensive based on arguments that the equilibrium distribution function cannot depend on how a system and bath are divided and that constraints must also be conserved quantities [36]. Briefly, we note that such requirements are well-known for equilibrium. The entropy must be a function, $S(U, V, N)$ of conserved quantities such as energy, volume, or particle number, in order to be a useful extremum principle. $S\left(U^{2}\right)$ has no value as a variational principle, because $U^{2}$ is not conserved in exchanges between states. Similarly, the constraints we use for dynamics are average fluxes, not higher cumulants like variances.

\section{APPENDIX B}

Here we give the details required to derive Eq. (12). For such three-cycles systems, we construct a transition matrix, $\mathbf{G}$, as follows:

$$
\mathbf{G}=\left(\begin{array}{lll}
\sigma_{00} & \sigma_{01} & \sigma_{02} \\
\sigma_{10} & \sigma_{11} & \sigma_{12} \\
\sigma_{20} & \sigma_{21} & \sigma_{22}
\end{array}\right),
$$

which is valid for any set of jump probabilities from site to site. From this transition matrix, we construct $Q$, as we had done in Eq. (4),

$$
Q=\left(\begin{array}{lll}
1 & 1 & 1
\end{array}\right) \mathbf{G}^{N}\left(\begin{array}{c}
\delta_{i, 0} \\
\delta_{i, 1} \\
\delta_{i, 2}
\end{array}\right) .
$$

To take the $N$ th power of $\mathbf{G}$, we first diagonalize $\mathbf{G}$. The diagonal $\mathbf{G}$ raised to the $N$ th power is then related to the $\mathbf{G}^{N}$ as follows:

$$
\mathbf{G}^{N}=\mathbf{S G}_{D}^{N} \mathbf{S}^{-1}
$$

where $S$ is the similarity transformation used to diagonalize the matrix. The eigenvalues and eigenvectors are too long to write out explicitly and found on MATHEMATICA. We use this 
form for $\mathbf{G}$ to compute the expressions given in Eq. (12). We calculate all this by taking the appropriate derivatives. To be explicit

$$
\langle J\rangle=\frac{\partial \ln Q}{\partial \ln \sigma_{10}}-\frac{\partial \ln Q}{\partial \ln \sigma_{01}}
$$

and

$$
\left\langle J^{2}\right\rangle_{c}=\frac{\partial^{2} \ln Q}{\partial \ln \sigma_{10}^{2}}+\frac{\partial^{2} \ln Q}{\partial \ln \sigma_{01}^{2}}-\frac{\partial^{2} \ln Q}{\partial \ln \sigma_{10} \partial \ln \sigma_{01}} .
$$

These expressions are too long to write out explicitly. On the other hand, the case we considered in this section were simple. This is because we set $\sigma_{00}=1-2 \sigma, \sigma_{11}=1-2 \sigma$, $\sigma_{22}=1-2 \sigma, \quad \sigma_{10}=\sigma+\epsilon, \quad \sigma_{20}=\sigma-\epsilon, \quad \sigma_{01}=\sigma-\epsilon, \quad \sigma_{21}=\sigma+\epsilon$, $\sigma_{02}=\sigma+\epsilon$, and $\sigma_{12}=\sigma-\epsilon$ after taking the derivatives. The simplified expressions are given in the main body. The curve for $\left\langle J^{2}\right\rangle_{c} /\langle J\rangle^{2}$ for the three cycle is plotted in Fig. 2.

\section{APPENDIX C}

Here we give more detail in deriving our general expression for the flux fluctuation ratio Eq. (20). The generality of the derivation that follows is required to verify that our flux fluctuation ratio does indeed take the form of Eq. (20) in any force regime for any state topology.

For ease of reference, we recall

$$
P\left(J_{i j} ; N \delta t\right)=Q^{\prime} / Q,
$$

where $Q$ in the above is $Q=\Pi_{m} q_{m}^{N_{m}}$ and $q_{m}$ is the dynamical partition function for state $m$ with $N_{m}$ particles initially. In addition, $Q^{\prime}$ is the sum over microtrajectories having net flux $J_{i j}$ between states $i$ and $j$. Specifically for the case of a single time-step, $N=1$, we have

$$
\begin{gathered}
Q^{\prime}=\prod_{m \neq i, j} q_{m}^{N_{m}} \sum_{l, p=0}^{N_{i}, N_{j}} \delta_{l-p, J_{i j}} \\
\left(\begin{array}{c}
N_{0} \\
l
\end{array}\right)\left(\begin{array}{c}
N_{1} \\
p
\end{array}\right) \sigma_{10}^{l} \sigma_{01}^{p} \sigma_{00}^{N_{0}-l} \sigma_{11}^{N_{1}-p} .
\end{gathered}
$$

In the above, we substitute the Kronecker delta, $\delta_{l-p, J_{i j}}$, by $\oint \frac{d z}{2 \pi i} z^{l-p-J_{i j}-1}$. This immediately returns,

$$
Q^{\prime}=\prod_{m \neq i, j} q_{m}^{N_{m}} \oint \frac{d z}{2 \pi i} z^{J_{i j}-1} \widehat{q}_{i}^{N_{i}} \widehat{q}_{j}^{N_{j}},
$$

where $\hat{q}_{i} \equiv q_{i}\left(\sigma_{j i} z^{-1}, \sigma_{i j} z\right)$. There is now an easy way of seeing that $P\left(J_{i j} ; N \delta t\right)=Q^{\prime} / Q$ will give a Gaussian for sufficiently large particle number. This is done by recognizing that $m(z) \equiv \hat{q}_{i}^{N_{i}} \hat{q}_{j}^{N_{j}}$ is the flux characteristic function and $c(z) \equiv \log (m(z))$ the corresponding cumulant generating function. A Gaussian is obtained by expanding the cumulant generating function in powers of $z$ (the variable conjugate to flux) to second order and carrying through with the integration. Higher order corrections are small when particle numbers, $N_{i}$ and $N_{j}$, are large enough.

To see this explicitly for a single time step, we substitute $z$ for $\exp (i \phi)$ in the previous expression. By dividing the resulting expression by $Q$, we find for $P\left(J_{i j} ; 1 \delta t\right)$

$$
P\left(J_{i j} ; 1 \delta t\right)=\frac{1}{q_{0}^{N_{0}} q_{1}^{N_{1}}} \int_{-\pi}^{\pi} \frac{d \phi}{2 \pi} e^{i J_{i j} \phi} e^{c(\exp (i \phi))} .
$$

Next, for large particle number, $c(\exp (i \phi))$ is expanded to second order around $\phi=0$. This yields

$$
c(\exp (i \phi)) \sim-i\left\langle J_{i j}\right\rangle \phi-\frac{1}{2}\left\langle J_{i j}^{2}\right\rangle_{c} \phi^{2}+\mathrm{O}\left(\phi^{3}\right) .
$$

The above was obtained by plugging in the explicit form for $c(\exp (i \phi))$ as $\log \left(\hat{q}_{i}^{N_{i}}\left(\sigma_{j i} e^{-i \phi}, \sigma_{i j} e^{i \phi}\right) \times \hat{q}_{j}^{N_{j}}\left(\sigma_{j i} e^{-i \phi}, \sigma_{i j} e^{i \phi}\right)\right)$. Expanding the latter in powers of $\phi$ it is recognized that the first- and second-order coefficients of $\phi$ are identical to those of Eqs. (7) and (8).

The coefficient of the third order term in $\phi$ is the skewness of the distribution which is small when $N_{i}$ and $N_{j}$ are large enough. Furthermore, since the integral quickly decays away from $\phi=0$, the limits of the integrand are extended from $-\pi$ to $\pi$ to $-\infty$ to $\infty$. In other words, we have

$$
P\left(J_{i j} ; 1 \delta t\right)=\int_{-\infty}^{\infty} \frac{d \phi}{2 \pi} e^{i J_{i j} \phi} e^{-i\left\langle J_{i j}\right\rangle \phi-1 / 2\left\langle J_{i j}^{2}\right\rangle_{c} \phi^{2}} .
$$

Upon integration, the above returns

$$
P\left(J_{i j} ; 1 \delta t\right)=\frac{1}{\sqrt{2 \pi\left\langle J_{i j}^{2}\right\rangle_{c}}} \exp \left[-\frac{\left(J_{i j}-\left\langle J_{i j}\right\rangle\right)^{2}}{2\left\langle J_{i j}^{2}\right\rangle_{c}}\right] .
$$

The ratio of $P\left(J_{i j} ; N \delta t\right)$ over $P\left(-J_{i j} ; N \delta t\right)$ for arbitrary time steps is presented in the main body as Eq. (20).
[1] M. Diez et al., Nat. Struct. Mol. Biol. 11, 135 (2004).

[2] D. R. Southworth and D. A. Agard, Mol. Cell 32, 631 (2008).

[3] M. Mickler et al., Nat. Struct. Mol. Biol. 16, 281 (2009).

[4] M. J. Rust et al., Science 318, 809 (2007).

[5] N. G. Van Kampen, Stochastic Processes in Chemistry and Physics (North-Holland, Amsterdam, 1981).

[6] P. B. Warren, S. Tănase-Nicola, and P. Rein ten Wolde, J. Chem. Phys. 125, 144904 (2006).

[7] E. T. Jaynes, in "Macroscopic Prediction," in Complex
Systems-Operational Approaches, edited by H. Haken (Springer-Verlag, Berlin, 1985), p. 254.

[8] E. T. Jaynes, in Annual Review of Physical Chemistry, edited by S. Rabinovitch (Annual Reviews, Palo Alto, 1980).

[9] M. Tribus and R. D. Levine, The Maximum Entropy Formalism (MIT Press, Cambridge, MA, 1981).

[10] G. Stock, K. Ghosh, and K. A. Dill, J. Chem. Phys. 128, 194102 (2008).

[11] K. Ghosh, K. A. Dill, M. Inamdar, E. Seitaridou, and R. Phil- 
lips, Am. J. Phys. 74, 123 (2006).

[12] P. Kienker, Proc. R. Soc. London, Ser. B 236, 269 (1989).

[13] P. B. Warren and J. L. Jones, Phys. Rev. Lett. 99, 108101 (2007).

[14] E. Seitaridou, M. Inamdar, R. Phillips, K. Ghosh, and K. Dill, J. Phys. Chem. B 111, 2288 (2007).

[15] D. Wu, K. Ghosh, M. Inamdar, H. J. Lee, S. Fraser, K. Dill, and R. Phillips, Phys. Rev. Lett. 103, 050603 (2009).

[16] H. Haken, Z. Phys. B: Condens. Matter 63, 505 (1986).

[17] R. M. L. Evans, Phys. Rev. Lett. 92, 150601 (2004).

[18] G. M. Wang, E. M. Sevick, E. Mittag, D. J. Searles, and D. J. Evans, Phys. Rev. Lett. 89, 050601 (2002).

[19] R. van Zon and E. G. D. Cohen, Phys. Rev. Lett. 91, 110601 (2003).

[20] U. Seifert, Phys. Rev. Lett. 95, 040602 (2005).

[21] F. Douarche, S. Joubaud, N. B. Garnier, A. Petrosyan, and S. Ciliberto, Phys. Rev. Lett. 97, 140603 (2006).

[22] D. Andrieux and P. Gaspard, J. Chem. Phys. 128, 154506 (2008).

[23] U. Seifert, EPL 70, 36 (2005).

[24] H. Qian and E. L. Elson, Biophys. Chem. 101-102, 565 (2002).
[25] R. D. Astumian, Proc. Natl. Acad. Sci. U.S.A. 104, 19715 (2007).

[26] A. Rákos and R. J. Harris, J. Stat. Mech.: Theory Exp. (2008) P05005.

[27] D. J. Evans, E. G. D. Cohen, and G. P. Morriss, Phys. Rev. Lett. 71, 2401 (1993).

[28] G. E. Crooks, Phys. Rev. E 61, 2361 (2000).

[29] D. Collin et al., Nature (London) 437, 231 (2005).

[30] G. Gallavotti and E. G. D. Cohen, Phys. Rev. Lett. 74, 2694 (1995)

[31] D. J. Evans and D. J. Searles, Adv. Phys. 51, 1529 (2002).

[32] D. M. Carberry, J. C. Reid, G. M. Wang, E. M. Sevick, D. J. Searles, and D. J. Evans, Phys. Rev. Lett. 92, 140601 (2004).

[33] E. T. Copson, Asymptotic Expansions (Cambridge University Press, Cambridge, England, 1965).

[34] J. E. Shore and R. W. Johnson, IEEE Trans. Inf. Theory 26, 26 (1980)

[35] J. W. Gibbs, Elementary Principles in Statistical Mechanics (Yale University Press, New Haven, CT, 1902), p. 33.

[36] "Information Theory and Statistical Mechanics," in Statistical Physics, edited by K. Ford (Benjamin, New York, 1963). 